relationships over time; that physical care tended to take priority over time spent exploring issues especially when time was limited. Reasons for not exploring and documenting were common for all staff groups.

Conclusions and recommendations The results were presented to the wider hospice team who made suggestions for change. These included: offering additional staff education and training in communicating with cognitively impaired people; proactively consulting informal carers to gain a deeper understanding of patients' needs; the use of a person-centred document for informal carers to complete with patients if able, that encouraged identification and exploration of psycho-social concerns; using routine handovers to highlight when these areas had not been addressed. A working group will be formed to implement and test changes to practice as part of the quality improvement in this area.

\section{EMERGENCY ADMISSIONS FROM CARE HOME TO HOSPITAL AT THE END OF LIFE: AN ANALYSIS OF NATIONAL DATA 2006-2015 FOR ENGLAND}

Rebecca Farndale, Stephen Barclay. Primary Care Unit, University of Cambridge

\subsection{6/bmjspcare-2018-ASPabstracts.117}

Objective Hospital admissions of care home residents close to the end of life are a source of concern and frequently described as 'inappropriate' and avoidable. However, the magnitude of this 'problem' on a national scale has not been investigated to date. This recently completed study investigated trends and characteristics of emergency admissions from care home to hospital where death occurred within seven days.

Methods Longitudinal and cross-sectional analysis of linked Hospital Episode Statistics with ONS mortality data for England 2006 to 2015: 185830 care home residents aged 25 +who experienced a last week of life emergency admission to hospital, with comparator group 903175 care home residents who died in care homes.

Results Of those in the last week of life in a care home $16.1 \%$ had an emergency hospital admission; of whom $91.3 \%$ died in hospital. Last week of life admissions contributed $14.6 \%$ of emergency admissions from care home to hospital and $9.2 \%$ of hospital mortality following emergency admission. Residents who had an emergency admission in the last week of life were less likely to be female (OR 0.84, CI: 0.83 to 0.85 ), from older age groups (OR 0.35 , CI: 0.34 to 0.37 , for $95+$ compared with 25-64) or to have dementia (OR 0.37 , CI: 0.36 to 0.37 ). They were more likely to have an underlying cause of death of stroke (OR 2.74, CI: 2.67 to 2.80), heart disease (OR 3.29, CI: 3.21 to 3.36), chronic respiratory disease (OR 3.91, CI: 3.81 to 4.01 ) acute respiratory disease (OR 5.74, CI: 5.61 to 5.88) or external injury (OR 9.73, CI: 9.32 to 10.16$)$ compared to cancer. Trend analysis revealed significant decreases in these admissions from 2008 onwards

Conclusions Last week of life hospital admissions are a small, and decreasing, risk for care home residents, the majority of whom remain in their care home in the final week of life. The implications for practice and policy are discussed

N.B. Data analysis recently completed: late-breaking abstract
91 HOW THE TRACEY JUDGEMENT HAS CHANGED DNACPR DISCUSSIONS IN PALLIATIVE CARE

Mike Macfarlane, Steph Shayler, Louisa Nelms, WM Cares, Derek Willis. West Midlands Deanery, Queen Elizabeth Hospital Birmingham, West Midlands Deanery, WM Cares, Severn Hospice

\subsection{6/bmispcare-2018-ASPabstracts. 118}

Background The decision published from the Court of Appeal of England and Wales in 2014 in the case of Tracey vs Cambridge University Hospital ('the Tracey judgement') changed the requirements for discussing Do Not Attempt Cardiopulmonary Resuscitation (DNACPR) decisions with patients.

Methods A retrospective case note review aiming to identify changes in practice when discussing DNACPR decisions following the Tracey judgement. 150 case notes from 2013 (before the Tracey judgement) were compared with 150 case notes from 2015 (following the Tracey judgement) at five hospices in the West Midlands. The notes were analysed to identify whether the judgement had resulted in a change in how frequently DNACPR decisions were discussed with patients or their families, as well as any changes in the rationale for not discussing such decisions.

Results Discussions with patients increased from 31\% to $60 \%$ and with relatives from $29 \%$ to $59 \%$. The most common reason that DNACPR decisions were not discussed with patients or relatives changed from a belief that these discussions would cause distress in 2013 (23\%) to the patient lacking capacity to engage in such discussions in 2015 (40\%). There was a lack of consistency and clarity in defining the concept of 'physical or psychological harm' following the Tracey judgement.

Conclusion Healthcare professionals specialising in palliative care in the region and time frame studied are discussing DNACPR decisions more frequently with patients and families following the Tracey judgement but clarity on what constitutes 'physical or psychological harm' caused by these discussions is still required.

\section{AN AUDIT OF CURRENT GUIDELINES AND RECOMMENDATIONS FOR MOUTH CARE IN PALLIATIVE AND END OF LIFE CARE}

Laura Daly, Yasmin Allen, Ashvir Basra, Jackie Sowerbutts, Mili Doshi, Samit Shah. Health Education UK

\subsection{6/bmjspcare-2018-ASPabstracts. 119}

Background Oral complications in palliative care patients are very common. Guidelines for oral care of these patients are available from several different sources within the UK. However, these guidelines are often inconsistent and it is unclear whether the recommendations are implemented in practice. This project aims to determine if the guidelines for oral care translate into the care provided to patients, and identify any additional techniques that are not published but provide benefits to patients in end of life care.

Methods A literature review of the guidelines and published evidence was conducted to collate a list of recommendations. A pilot survey and qualitative research was conducted with Special Care Dentistry Consultants, Palliative Care consultants, geriatricians, and those providing day-to-day care for palliative care patients. This focused on what treatment was being provided to maintain oral care and comfort for palliative care patients. 
Results Data was collected from a large range of health professionals working with palliative care patients. It was found that approximately $34 \%$ of respondents were not aware of any oral care guidelines for palliative care patients. Those that did were mostly aware of the NICE guidelines.

There was variation between all respondents as to what treatment was routinely given for various oral conditions. Some practices restricted in certain trusts were shown to be used elsewhere, for example foam swab use (70\%), which is not routinely recommended by dental professionals.

Although the majority of respondents reported their patients have personalised oral care plans in place, $43 \%$ do not.

Conclusions Clearer and more concise guidelines for oral care in palliative care patients is needed to ensure staff are able to offer the same level of evidence-based care consistently across the UK. The importance of oral care plans for these patients should be promoted, to ensure patients have continuity of care.

\section{PATTERNS OF GENERAL PRACTICE PRESCRIBING IN THE LAST YEAR OF LIFE}

${ }^{1}$ Amelia Harshfield, ${ }^{2}$ Rupert Payne, ${ }^{1}$ Stephen Barclay. 'University of Cambridge; ${ }^{2}$ University of Bristol

\subsection{6/bmispcare-2018-ASPabstracts. 120}

Background Little is known about patterns of prescribing in the last year of life in primary care.

Methods Recently completed analysis of UK Clinical Practice Research Datalink (CPRD), a large database extracted from GP records that is representative of the UK population. Prescribing data for the last 12 months of life were extracted from 118571 patients $^{6}$ GP records who died between September 2010 and August 2015. Two clinicians used British National Formulary codes to identify eight palliative care drug categories: antiemetics, anti-secretory, benzodiazepines, steroids, strong injectable opioids, strong patch opioids, strong enteral opioids, and weak oral opioids. The percentage of patients prescribed each medications was calculated at weekly intervals during the year before death, further stratified by cause of death and age at death. Random effects logistic regression models were run adjusted for age-group, sex, and cause of death.

Results Levels of prescribing significantly increased for all drugs at time of death compared with one year before death (with the exception of weak oral opioids and steroids where results were inconsistent across ages, genders, and causes of death). The largest increase was for $90+$ year old male patients prescribed strong injectable opioids (OR: 21032, 95\% CI: 3499 to 12641) and the greatest decrease for females prescribed steroids who died of external causes (OR: 0.52, 95\% CI: 0.34 to 0.70 ). Patients who died of cancer generally showed the most marked increase in prescribing towards the end of life compared with other causes of death.

Conclusions Primary care patterns of prescribing in the last year of life has not been previously investigated. The findings of this recently completed study of a large nationally-representative dataset reveal increasing GP prescribing in response to the rising symptom burden of all patient groups as death approached. The implications for clinical practice and future research will be discussed.
94 GUIDELINE DEVELOPMENT FOR THE MANAGEMENT OF AGITATION IN THE LAST WEEKS OF LIFE

Kate Nolan, Helen Bonwick, Claire Robinson, Mark Mills, A Griffiths, P Shepherd, N Shah. Marie Curie Hospice Liverpool

\subsection{6/bmjspcare-2018-ASPabstracts.121}

Background Agitation is a distressing and common symptom at the end of life; despite this the evidence for the management of this symptom is poor.

Aim

- Audit clinical practice around management of terminal agitation with patients and healthcare professionals (HCPs) against regional standards.

- Use audit outcomes to update regional standards and guidelines

\section{Method}

- Systematic literature review examining the evidence base.

- Survey of HCPs working in specialist palliative care.

- Retrospective case note review of patients with a diagnosis of terminal agitation receiving specialist palliative care in hospital, hospice or community settings.

Results Below is a summary from 185 responses from 11 different healthcare settings in the Merseyside area from retrospective case note review:

- Hospice 54.5\%, Hospital 29.7\%, Home 11.8\%, other including Nursing homes 3\%.

- Main reversible causes documented included: pain; breathlessness: constipation and urinary retention.

- $94 \%$ of patients had medications reviewed.

- Non-pharmacological interventions formed part of the overall management plan.

- $82 \%$ of patients had psychological and spiritual support offered to the patient and or family.

- First line pharmacological intervention is Midazolam, Levomepromazine is second line.

- In 92\% of cases a Syringe driver (CSCI) was used.

- Majority of deaths occurred in 3 days or less

- Communication with patient and family formed a key theme. At the time of death:

- Midazolam prescribed in 146 cases (mean $26 \mathrm{mg}$ - range 0$60 \mathrm{mg}$ ).

- Levomepromazine prescribed in 61 cases (Mean $56.8 \mathrm{mg}-$ range $5-250 \mathrm{mg}$ ).

- Haloperidol 25 cases (Mean $3.5 \mathrm{mg}$ - range 1-8 mg).

- Phenobarbital 9 cases ( Mean 922 mg - range 200-1200 mg).

Conclusion This Audit revealed the challenges and wide variation in practice with regards to management of terminal agitation. These findings have informed an update of the regional palliative care guidelines.

\section{THE 'SUPPORT OF CARE CYCLE': INTEGRATING ETHICS INTO HEALTHCARE PROFESSIONAL SUPPORT AND DEVELOPMENT IN HOSPICES}

Craig Gannon. Princess Alice Hospice

10.1136/bmjspcare-2018-ASPabstracts. 122

Background High-quality hospice care is dependent on an expert caring and resilient workforce who can cope with the 